\title{
A Review on Recognition Rate Techniques for 2-D and 3- D Image
}

\author{
Navneet Kaur \\ M. Tech Student, Department of ECE \\ Guru Nanak Dev University, Amritsar
}

\author{
Jasdeep Kaur \\ Asst. Prof., Department of ECE \\ Guru Nanak Dev University, Amritsar
}

\begin{abstract}
Machine recognition of faces is a biometric process in which face of a person is recognized by comparing the present image of a person with the image already present in the database. Demand is increasing rapidly as recognition is a vigorous research issue because of its non-copier characteristic. Compelling attention has been received by this technology because it has potential for tremendous applications like criminal identification, bank/store security, credit card verification, healthcare, marketing, automatic attendance etc. Face recognition is very secure method but its performance is degraded by some factors. Several researchers have recommended methods to nullify the effects of these factors. This paper provides a review on some effective $2 \mathrm{D}$ and $3 \mathrm{D}$ face images techniques with pose variations which are compared on the basis of recognition rates. From the discussed 2D face images techniques, recognition rate up to $100 \%$ was obtained by Kernal Canonical Correlation analysis (KCCA) only if input images are less than 200 images. If input images are more than 200 then 2D image based approach has higher recognition rate and is also simpler. From the discussed 3D techniques, recognition rate is highest of morphable model and also this technique is not affected by occlusion.
\end{abstract}

\section{Keywords}

Face recognition, 2D techniques, 3D techniques, Recognition rates

\section{INTRODUCTION}

Disciplines such as Pattern recognition, image processing, neural networks etc. are some in which machine recognition of faces is emerging today. Psychologically, a rich source of information about human behaviour is face. Face is one of the important parts of human body from which the person is recognized by humans but machine recognition is a difficult task as machine can recognize the particular person by matching the current photo of a person which is taken with the help of a camera with the photograph database. Face recognition is the example of biometric systems [1]. A term for body calculations and measurements is known as biometric and refers to metrics which are related to characteristics of humans. In recent years, one of the auspicious systems which have been transpired to recognize faces or to secure data is biometric based techniques [2]. Fingerprint geometry, iris pattern, signature, retinal patterns, voice pattern, face etc. are various physiological properties which are being used for identification. All these biometrics are being captured and compared with the stored data to identify the person. Cooperation from person is required in most biometric techniques but being passive and natural, face recognition has clear advantage over other techniques. Out of all these biometrics, face recognition is the most attractive biometrics to secure data because of its non-invasive nature as cooperation of a person is not needed and without coming in notice a face is recognized in any erratic situation and uncontrolled environment $[3,23]$. In previous years Certification and access of people physically and virtually depends on passwords, token no., smart cards etc. and these methods examine individual behavioural or physiological characteristics to determine individual identity which was a very difficult and time consuming process but nowadays biometric based techniques are the most promising and easy option to recognize individual person [2]. Florida (state of US) was the first where the large scale application of face recognition was effectuated. Access control, surveillance system, image database identification, credit card verification, seizure control, healthcare, marketing, automatic attendance etc. are some of the applications of face recognition [2]. For the recognition of face, a typical process needs to be followed.

\subsection{Process of face recognition}

Machine has to undergo a long process to recognize a person. For this, digital camera captures the image, and machine tries to recognize the face of a person by analysing the characteristics of image in which mouth, jaw edges, nose, distance between eyes got analysed. These measurements are included and when comparison starts, these are used for comparison. Basic recognition process requires the accomplishment of three steps: Face Detection, Feature Extraction and Face Recognition. Basic block diagram and demonstration of these steps are discussed below.

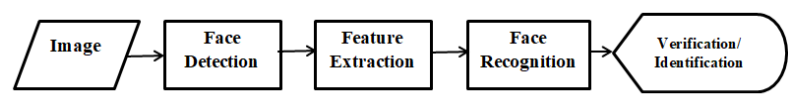

Figure 1-Face recognition process

\subsubsection{Face Detection}

Face detection is the second step after an image is captured by the camera. The main motive of this step is to search in an image where human faces appear and locate. After this step, patches are visible on each face of an image, which comes as an output [4].

\subsubsection{Feature Extraction}

Output image of face detection step can't be directly used because of its large size due to which FR (face recognition) system becomes difficult to build. So, feature extraction step is important which in an image reduces the dimensions, cleans the noise. From the image containing patches which depicts the face of a person, face components such as eyes, nose mouth etc. are localised with the help of with the help of feature extraction $[4,5]$. 


\subsubsection{Face Recognition}

The final step of FR process is face recognition. For this step, firstly face database is essential to be build which consists of many images of one person which was already captured. Then the features of present image which are acquired in previous two steps are compared with database images [4].

2D images were used in face recognition software [16-19] in which 2D images was compared with the database images which were also 2D. The person is supposed to look directly at the camera so that software gives accurate results. But most of the images were not in the desired manner of the software requirement. May be person is not looking directly to camera or location gets suffered by poor lighting. These factors affect the performance of the system [6]. Present automated face recognition systems have reached a certain level of maturity, even though there are such factors which limits the performance of face recognition systems. These factors are imposed by many real applications. For example, pose of a person changes or illumination changes in outdoor or uncontrolled environment or any facial mark etc. are the factors which affect the performance of an automated face recognition system.

Expression of a person, significant pose and illumination differences are the main points from which 3D technology begins. It is expected to develop more realistic 3D face recognition with the construction of bigger $3 \mathrm{D}$ face databases that contain enough samples for different illumination, pose, and expression variations [7]. So, that a person can be detected from any direction or even if a person is in different poses. Two prominent issues for appearance- or image-based approaches are the illumination and pose problems. Many approaches have been proposed to handle the above mentioned issues [8].

\subsection{Factors affecting the performance of face recognition algorithm}

\subsubsection{Occlusion}

When a face of a person is covered with some other object like a piece of a cloth, sunglasses etc. it is known as occlusion as shown in figure 1.2. The performance of the face recognition algorithm gets affected when a face is occluded because system faces difficulty in recognition when face of a person is not properly captured by the camera [9].

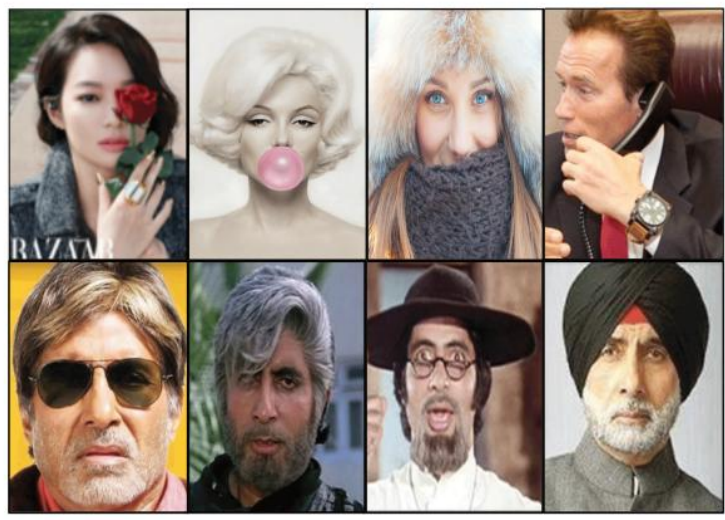

Figure 2- Occluded faces

\subsubsection{Illumination}

Illumination means some parts of the face are invisible due to change in lighting so it becomes difficult for an algorithm to recognize a face. But today, researchers are inventing new techniques which can nullify the effects of illumination completely. One such technique was suggested in "An Automatic Face Identification System Using flexible Appearance Models" by Anees Buzdar and Hammad Khan [10] which is local binary pattern.

\subsubsection{Pose}

Sometimes when people are not facing the camera then it became a big obstacle in the path of recognition algorithm as shown in figure 1.3. Even sometimes it becomes very difficult to recognize images of same people with different poses as compared to images of two different people with same pose. So pose difference plays an important role in this process [11].
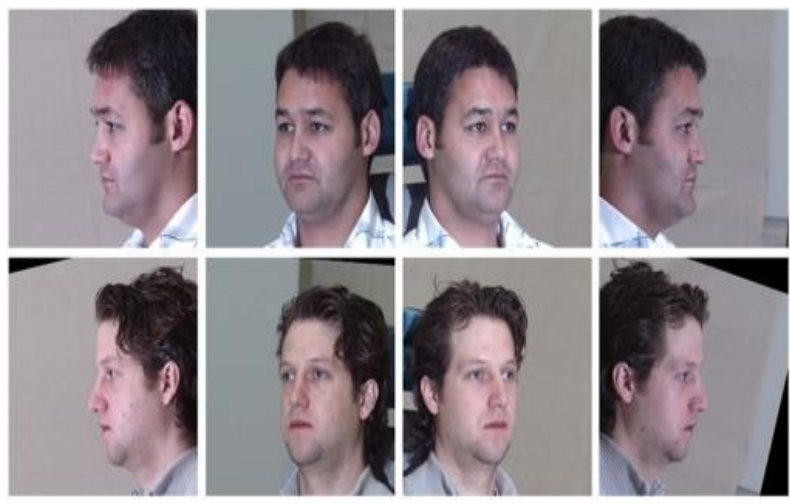

Figure 3 - Pose variations

This review paper consists of three categories of face recognition techniques. In first category there are only general algorithms which do not peculiarly handle the pose variation but designed for conventional face recognition which are described in section 2. While in other two categories, techniques specifically handle pose variations which are discussed in section 3. Section 3.1 elaborates 2D pose variation techniques and $3 \mathrm{D}$ pose variation techniques are discussed in section 3.2. On the basis of recognition rates all these techniques are compared in section 4 .

\section{FACE RECOGNITION TECHNIQUES}

Face recognition is becoming a hot topic in industry now-adays. Day by day researchers are proposing new techniques to increase the capability of the system to recognize the face. Researchers have proposed face recognition techniques which are:

\subsection{Joint Sparse Coding}

Vishal M. Patel et al. [12] for robust recognition at a low resolution, they proposed a technique known as joint sparse coding. Two datasets such as challenging outdoor face dataset and standard datasets were used to demonstrate the effectiveness of proposed method. Results show that as compared to other competitive low resolution recognition algorithms, the proposed method was efficient and could perform better

\subsection{Coloured Images}

Joonwhoan Lee and Deepak Ghimire [13] described a new method of detecting human face in coloured images. Entire image for the presence of face was scanned by a technique like window based classifier which was used by many systems that exist for face recognition. Technique based on information of skin and edge tone of coloured images known as lighting insensitive face detection was described. In that method if the input image was from unconstrained 
illumination condition then image enhancement i.e. images in different lighting was first converted into uniform lighting conditions. Then skin segmentation was conducted in RGB and $\mathrm{YCbCr}$ space. The results were refined by method known as skin tone percentage index. Analyzation of connected components was done by primitive shape features and the standard deviation of human face area in the corresponding grayscale image of skin and edge segmented area.

\subsection{Sparse representation based classification (SRC)}

Meng Yang and Lei Zhang [14] had successfully used SRC for face recognition by coding input testing image as a sparse linear combination of training samples via 11-norm minimization. SRC could lead to robust face recognition results against occlusion by introducing an identity occlusion dictionary to sparsely code occluded portions in human face images. Sparse coding was very expensive because of the large amount of atoms in occlusion dictionary. For SRC, Gabor-features were used which was presented in this paper. Occlusion dictionary became compressible with the use of Gabor Kernels and after that algorithm computed by Gabor occlusion dictionary were presented. In this Gabor occlusion dictionary, number of atoms was reduced due to which cost in coding the occluded face images was reduced.

\subsection{Hierarchical MRF}

Shervin Rahimzadeh Arashloo and Josef Kittler [15] proposed an image matching method which was based on hierarchical MRF in which pixel-wise correspondences were found between images which were viewed from different angles. Pair of facial images was also densely registered with the help of this method. CMU PIE database was used to classify this method.

\subsection{Elastic Bunch Graph matching}

Jean-MarcFellous et al. [16] proposed a system which recognizes faces from single images out of large database which contained one image of each person. By extracting concised face descriptions in the form of an image graph, the system collapsed most of variance. Set of wavelet components (jets) were used to describe the fiducial points on the face like eyes, mouth etc. The graph representation of a face is based on the Gabor wavelet transform, a convolution with a set of wavelet kernels.

\subsection{Hidden markov model}

Ara V. Nefian and Monson H. Hayes III [17] described a method using the coefficient of Karhunen Loeve Transform (KLT); observation vectors which were used to characterize the states of hidden markov model were obtained. A recognition rate was slightly improved and computational complexity of the previous hidden markov model based face recognition was reduced by the face recognition system introduced in this paper. Stable with the hidden markov model of face, novel hidden markov model based face detection was introduced, which uses feature extraction techniques same as face recognition. Advantages of this method are flexible, more efficient framework, accurate in variations in lighting conditions, complexity efficiency.

\section{POSE VARIATION FACE RECOGNITION}

It is easy to detect a face if a person is facing the camera but if the person in under defective lighting i.e. if the face is illuminated or occluded or if there are pose variations then it becomes difficult for a system to recognize a face. These effects are degrading the performance of face recognition systems. So, now the researchers are trying to nullify these effects. Researchers had proposed some techniques for nullification of pose variations. $2 \mathrm{D}$ and $3 \mathrm{D}$ techniques are used to handle this affect. 3D or 2D images are the only type of facial information on which almost every face recognition technique relies [18].

\subsection{D Techniques}

Today everyone is concentrating on $3 \mathrm{D}$ based face recognition but there are still some areas for e.g. CT scan in which 2D based recognition is better [19]. Some of 2D techniques have even better recognition rates than $3 \mathrm{~d}$ techniques. This can be seen from recognition rates of following techniques [Table-1, 2].

\subsubsection{Kernel canonical correlation analysis ( $K C C A)$}

Malek Nadil et al. [20] proposed a method for identification of profile face images which was based on mapping of $2 \mathrm{D}$ face images. Profile face images were changed into frontal face images which were issued by KCCA. FERET database was used to perform tests through which the effectiveness of the approach was shown. Leave one out like protocol (LOOLP) was used for tests. Results of test show that this technique was better than other 2D techniques. They were only restricted to the main components of the face like eyes, chin, mouth, nose because between profile pose and frontal pose, there was a non-linearity of transformation.

\subsubsection{Moment based feature extraction} techniques (Hu's Moments and Zernike Moments) Jagdish P. Sarode and Alwin D. Anuse [21] proposed model based approach in which feature extraction technique which was based on moments( Zernike Moments and Hu's Moments) was implemented on two face images having different poses. KNN classifier was used to classify the extracted features. Recognition Rate was also calculated. 4th order Zernike moments and 8th order Zernike moments and $\mathrm{Hu}$ moments through which the features were extracted, were used to conduct the experiments. Using 8th order Zernike moments, recognition rate was $78.09 \%$, using $\mathrm{Hu}$ moments rate was $31.03 \%$ and with $4^{\text {th }}$ moment Zernike, it was $78.44 \%$ was observed. These shows that Zernike moment were good for recognition as compared to Hu's moment.

\subsubsection{Stacked Progressive Auto-Encoders (SPAE)}

Meina Kan et al. [22] invented Stacked Progressive AutoEncoders (SPAE) and advised to learn robust face features to solve the problem of pose variations. Then for step by step reduction of pose variations, non-frontal face images had to be converted into frontal face images which would be accomplished with the help of stacking this shallow progressive auto encoder. As a consequence, they got that there was very little pose variations in the result of uppermost hidden layer which they could use as face recognition's robust face feature. In this approach, test images did not require any pose estimation which was an interesting feature.

\subsubsection{Probabilistic Elastic Matching (PEM)}

Haoxiang Li et al. [23] proposed the PEM method to nullify the effects of different poses. In which multi-scale image 
patches which was densely sampled was used to extract the local features. In training corpus, spatial appearance distribution of all face images was captured to train the Gaussian mixture model (GMM). Location terms and appearance's influence was balanced by confining GMM's each mixture component to be a spherical Gaussian. To know whether there was a match between the face tracks or not, they trained SVM on the vector integrating the feature pairs' difference vectors for verification. Accuracy was improved when the face tracks modelled the pose variations between them for which trained GMM was adapted by the Bayesian adaption algorithm. Based on youtube faces dataset, benchmark datasets and most restricted LFW, proposed method showed better results than the state-of-the-art methods.

\subsubsection{D image-based approach}

Sang-Il Choi et al. [24] suggested a method in which both pose and illumination problems were handled simultaneously. Recognition rate were better in this as compared to 3D techniques. Feature extraction method was used for each pose class to construct a feature space. Pose class for an image was determined firstly with the help of the pose estimation technique then for every pose class, shadow compensation procedure was carried on for compensating every image. After this, for identification these images were used with the help of classification rule. Recognition rate with the help of this technique is $99 \%$.

\subsection{D Techniques}

To tackle with pose and illumination variations one of the eminent approach is face recognition with $3 \mathrm{D}$ images. Human heads are $3 \mathrm{D}$ objects and its all viewpoints are also in $3 \mathrm{D}$ spaces due to which this approach overpower when it comes to handle pose variations [11].

\subsubsection{Support Vector Machines}

Shaogang Gongb et al. [25] proposed a method in which segments were used to separate view sphere and face detector was used on each segment. There was no information whether the image was face or not even then researchers estimated the pose of an image. Support vector regression was used to construct the pose estimator. This was done so that an appropriate face detector was used which determined that an image was a face. Computational efficiency was achieved with this method. Because of the construction of detector on small range of views, accuracy was achieved. Combination of both SVM and Eigen face methods was used for detection with improved speed. This combination suppressed the disadvantages of both method and kept the advantages that is speed from Eigen face and accuracy from SVM.

\subsubsection{Based on Fitting a 3D Morphable Model}

Volker Blanz and Thomas Vetter [26] presented a paper in which pose and illumination variations were studied. To handle these variations, an algorithm was studied in which by using computer graphics the 3D space image formation was processed. From single image, texture and shape of the image was also estimated and which was achieved by fitting 3D faces morphable model to images. With the help of set of textured 3D scans of heads, this model was learned. Morphable model's construction, algorithm and face identification's framework was described by them in this paper. They also presented results with FERET database's 1940 images and with 4488 images from CMU-PIE.

\section{Recognition Rate of 2D and 3D Techniques}

Recognition is the act of recognizing someone or identifying something when you see it. Recognition rate is the percentage which shows how correctly an image is identified by the system. Mathematically,

Recognition rate $=$ (no. of correctly identified images $/$ Total no. of images) $* 100$

Below table exhibits the database used by the $2 \mathrm{D}$ and $3 \mathrm{D}$ techniques and their respective recognition rates

Table 1- Recognition rate (\%) of 2D techniques [20-24]

\begin{tabular}{|c|c|c|c|}
\hline Technique & \multicolumn{2}{|l|}{ Database } & $\begin{array}{l}\text { Recognition } \\
\text { rate }(\%)\end{array}$ \\
\hline \multirow{2}{*}{$\begin{array}{l}\text { Kernel } \\
\text { canonical } \\
\text { correlation } \\
\text { analysis } \\
\text { (KCCA) }\end{array}$} & \multicolumn{2}{|c|}{ FERNET 200 people } & 100 \\
\hline & \multicolumn{2}{|c|}{ FERNET 600 people } & 76.5 \\
\hline \multirow{3}{*}{$\begin{array}{l}\text { Moment based } \\
\text { feature } \\
\text { extraction } \\
\text { techniques }\end{array}$} & $\begin{array}{l}\text { Hu's } \\
\text { moment }\end{array}$ & $\begin{array}{l}\text { IIT } \\
\text { (female) }\end{array}$ & 27.27 \\
\hline & $\begin{array}{l}4^{\text {th }} \text { order } \\
\text { Zernike }\end{array}$ & $\begin{array}{l}\text { IIT } \\
\text { (female) }\end{array}$ & 86.36 \\
\hline & $\begin{array}{l}8^{\text {th }} \text { order } \\
\text { Zernike }\end{array}$ & $\begin{array}{l}\text { IIT } \\
\text { (female) }\end{array}$ & 80.30 \\
\hline \multirow{2}{*}{$\begin{array}{l}\text { Stacked } \\
\text { progressive } \\
\text { auto encoders } \\
\text { (SPAE) }\end{array}$} & \multicolumn{2}{|l|}{ FERNET } & 92.5 \\
\hline & \multicolumn{2}{|l|}{ Multipie } & 91.4 \\
\hline \multirow{2}{*}{$\begin{array}{l}\text { Probabilistic } \\
\text { elastic } \\
\text { Matching }\end{array}$} & \multicolumn{2}{|c|}{ Youtube base face } & 77.52 \\
\hline & \multicolumn{2}{|l|}{ LFW } & 81.10 \\
\hline \multirow{2}{*}{$\begin{array}{l}\text { 2D image based } \\
\text { approach }\end{array}$} & \multicolumn{2}{|c|}{ CMU-PIE } & 99 \\
\hline & \multicolumn{2}{|l|}{ Yale B } & 94.8 \\
\hline
\end{tabular}

From the table 1, it is seen that KCCA has highest recognition rate in $2 \mathrm{D}$ techniques of number of input images is not more than 200. If number of images is more than 200 then 2D based approach is best and this technique is also simpler. Similarly, recognition rate for $3 \mathrm{D}$ techniques for different database can also be found. Given below are the recognition rates of 3D techniques.

Table 2- Recognition rate (\%) of 3D techniques [25, 26]

\begin{tabular}{|l|l|l|}
\hline Technique & Database & $\begin{array}{l}\text { Recognition rate } \\
(\mathbf{\%})\end{array}$ \\
\hline $\begin{array}{l}\text { Based on fitting } \\
\text { the } \\
\text { morphable model }\end{array}$ & CMU-PIE & 95 \\
\cline { 2 - 3 } & FERET & 95.9 \\
\hline $\begin{array}{l}\text { Support vector } \\
\text { machines }\end{array}$ & Live video & 93 \\
\hline
\end{tabular}


From table 2, it is seen that among 3D techniques, morphable model is good and also this method is not affected by occlusion but speed is more in support vector machines.

Every technique has some advantages and disadvantages. Year, advantages and disadvantages of $2 \mathrm{D}$ and $3 \mathrm{D}$ face recognition techniques are summarised below.

Table 3- Advantages and disadvantages of $2 \mathrm{D}$ and $3 \mathrm{D}$ pose variation techniques [18-25]

\begin{tabular}{|c|c|c|c|}
\hline $\begin{array}{l}\text { Pose } \\
\text { variation }\end{array}$ & Year & Advantages & Disadvantages \\
\hline $\begin{array}{l}\text { Kernel } \\
\text { canonical } \\
\text { correlation } \\
\text { analysis } \\
\text { (KCCA) }\end{array}$ & 2017 & $\begin{array}{l}-100 \% \\
\text { identification } \\
\text { on rate up to } \\
200 \text { people }\end{array}$ & $\begin{array}{l}\text { Identification } \\
\text { rate decrease if } \\
\text { number of } \\
\text { people increase }\end{array}$ \\
\hline $\begin{array}{l}\text { Moment } \\
\text { based } \\
\text { feature } \\
\text { extraction } \\
\text { techniques }\end{array}$ & 2014 & $\begin{array}{l}\text { - } 78 \% \\
\text { recognition } \\
\text { rates with } \\
\text { Zernike } \\
\text { moments }\end{array}$ & $\begin{array}{l}\text { Recognition } \\
\text { decreases with } \\
\text { Hu's moment }\end{array}$ \\
\hline $\begin{array}{l}\text { Stacked } \\
\text { progressive } \\
\text { auto } \\
\text { encoders } \\
\text { (SPAE) }\end{array}$ & 2014 & $\begin{array}{l}\text { - } \text { No pose } \\
\text { estimation } \\
\text { required } \\
\text { - Superior to } \\
\text { existing 2D } \\
\text { methods }\end{array}$ & \\
\hline $\begin{array}{l}\text { Probabilisti } \\
\text { c elastic } \\
\text { Matching }\end{array}$ & 2013 & $\begin{array}{l}\text { - Improved } \\
\text { accuracy }\end{array}$ & \\
\hline $\begin{array}{l}\text { Based on } \\
\text { fitting the } \\
\text { 3D } \\
\text { morphable } \\
\text { model }\end{array}$ & 2003 & $\begin{array}{l}\text { - Performance } \\
\text { not affected by } \\
\text { occlusion } \\
\text { - } 95 \% \text { correct } \\
\text { identification }\end{array}$ & \\
\hline $\begin{array}{l}\text { Support } \\
\text { Vector } \\
\text { Machine }\end{array}$ & 2004 & - More accuracy & \\
\hline $\begin{array}{l}2 \mathrm{D} \text { image } \\
\text { based } \\
\text { approach }\end{array}$ & 2011 & $\begin{array}{l}\text { - } \text { High } \\
\text { recognition } \\
\text { - simpler }\end{array}$ & \\
\hline
\end{tabular}

\section{CONCLUSION}

In this paper, comparative analysis of existing $2 \mathrm{D}$ and $3 \mathrm{D}$ techniques was discussed. Recognition rate was a factor on the basis of which all techniques were compared. Analysis showed that out of the discussed 2D techniques, Kernel Canonical Correlation Analysis (KCCA) had highest recognition rate. But KCCA will be at highest position only if number of inputs is less than 200. If number of input images increases then performance decreases to $76.5 \%$. Other than this, 2D based approach was best with more number of inputs and also this technique was simpler. From the discussed 3D techniques, Morphable model had high recognition rate as compared to support vector machines. Also performance of morphable model was not affected by occlusion. Advantages and disadvantages of all techniques were discussed in this paper.

\section{REFERENCES}

[1] R. Chellappa, C.L. Wilson, and Sirohey, "Human and Machine Recognition of Faces, A survey," Proc. of the IEEE, Vol. 83, pp. 705-740 (1995)

[2] Divyarajsinh N. Parmar, Brijesh B. Mehta "Face Recognition Methods \& Applications" International Computer Technology \& Applications (IJCTA), Vol 4, pp. 84-86 (2013)

[3] R.Rajalakshmi, Dr.M.K.Jeyakumar "A review on classifiers used in face recognition methods under pose and illumination variations" IJCSI International Journal of Computer Science Issues, Vol. 9, pp 474-485 (2012)

[4] Wei-Lun Chao "Face Recognition" GICE, National Taiwan University (2007)

[5] Paola Campadelli, Raffaella Lanzarotti and Giuseppe Lipori "Automatic Facial Feature Extraction for Face Recognition” pp.558 (2007)

[6] Dr.S.B.Thorat, S.K.Nayak, Miss. Jyoti P Dandale "Facial Recognition Technology: An analysis with scope in India" International Journal of Computer Science and Information Security (IJCSIS), Vol. 8, pp. 325-330, (2010)

[7] Bernd Heisele, Purdy Ho, Tomaso Poggio "Face Recognition with Support Vector Machines: Global versus Component-based Approach" International Conference on Computer Vision (2001)

[8] A. S. Tolba, A.H. El-Baz, and A.A. El-Harby "Face Recognition: A Literature Review" International Journal of Signal Processing Vol 2 (2006)

[9] Thazheena, Aswathy Devi "A review on face detection under occlusion by facial accessories" International Research Journal of Engineering and Technology (IRJET) Volume: 04, pp 672-674 (2017)

[10] A.Lanitis, C.J.Taylor and T .F .Cootes "An Automatic F ace Identification System Using Flexible Appearance Models" Image and Vision Computing, volume 13, pages 393-401 (1995)

[11] Xiaozheng Zhang, Yongsheng Gao "Face recognition across pose: A review" Pattern Recognition volume 42, pp 2876-2896 (2009)

[12] Sumit Shekhar, Student Member, IEEE, Vishal M. Patel, Member, IEEE, and Rama Chellappa, Fellow, IEEE, "Synthesis-based Robust Low Resolution Face Recognition" IEEE transactions on information forensics and security (2017)

[13] Joonwhoan Lee and Deepak Ghimire "A Robust Face Detection Method Based on Skin Color and Edges" Journal of Information Processing Systems, Vol.9, pp 141-156 (2013)

[14] Meng Yang, Lei Zhang "Gabor Feature based Sparse Representation for Face Recognition with Gabor Occlusion Dictionary" European Conference on Computer Vision ECCV, vol 6316, pp 448-461 (2010) 
[15] S. R. Arashloo, and J. Kittler, "Hierarchical Image Matching for Pose-invariant Face recognition," British Machine Vision Conference (BMVC) (2009)

[16] Laurenz Wiskott, Jean-Marc Fellous , Norbert Kru“ger, and Christoph von der Malsburg "Face Recognition by Elastic Bunch Graph Matching" In Intelligent Biometric Techniques in Fingerprint and Face Recognition, publ. CRC Press, , pp. 355-396 (1999).

[17] Ara V. Nefian and Monson H. Hayes III "hidden markov model by face detection and recognition" ICASSP98, vol.5, pp.2721-2724 1(1998)

[18] Yingjie Wang, Chin-Seng Chua, Yeong-Khing Ho "Face Recognition from 2D and 3D Images" International Conference on Audio- and Video-Based Biometric Person Authentication, vol 2091, pp 26-31, (2001)

[19] Vijay H Mankar "A review on 2D, 2.5D and 3D image visualization techniques" International Journal of Advanced Research in Computer Engineering \& Technology (IJARCET) Volume 5 (2016)

[20] Malek Nadil, Feryel Souami, Abdenour Labed and Hichem Sahbi "KCCA- based technique for profile face identification" Nadil et al. EURASIP Journal on Image and Video Processing DOI 10.1186/s13640-016-0123-8 (2017)

[21] Jagdish P. Sarode and Alwin D. Anuse "Face Recognition under Pose Variations" (IJCSIT) International
Journal of Computer Science and Information Technologies, Vol. 5, pp 2689-2693 (2014)

[22] Meina Kan, Shiguang Shan, Hong Chang, Xilin Chen "Stacked Progressive Auto-Encoders(SPAE) for Face Recognition Across Poses" IEEE Conference on Computer Vision and Pattern Recognition (CVPR), pp. 1883-1890 (2014)

[23] Haoxiang Li, Gang Hua, Zhe Lin, Jonathan Brandt, Jianchao Yang "Probabilistic Elastic Matching for Pose Variant Face Verification" Computer Vision and Pattern Recognition (CVPR), IEEE Conference, pp 3499-3506, (2013)

[24] Sang-Il Choi, Nojun Kwak, Chong-Ho Choi "Face recognition based on $2 \mathrm{D}$ images under illumination and pose variations" Pattern recognition letters, volume 32, pages 56157, DOI: 10.1016/j.patrec.2010.11.021 (2011)

[25] Yongmin Lia, Shaogang Gongb, Jamie Sherrahc, Heather Liddell "Support vector machine based multi-view face detection and recognition" Image and Vision Computing, vol 22, pp 413-427, DOI:10.1016/j.imavis.2003.12.005 (2004)

[26] Volker Blanz and Thomas Vetter "Face Recognition Based on Fitting a 3D Morphable Model", IEEE transactions on pattern analysis and machine intelligence, vol. 25, pp 10631074, DOI: 10.1109/TPAMI.2003.1227983, (2003) 